man trials ${ }^{2 . .3}$ inevitably reinforced doubts that PFCs would be useful or even desirable.

There have been two main developments in this area during the past 5 years. The first is improvement of the oxygen-carrying capacity, by the development of second-generation, highphase-volume (greater than 50\%) emulsions with excellent stability at room temperature and reduced toxic effects due to the use of purified components 5 . Second, PFCs and their emulsions have been used in a broad range of applications, for example, organ perfusion and preservation, diagnosis, cancer therapy, ophthalmology and cell cultures ${ }^{2,3}$.

We propose a further therapeutic use for PFC emulsions, as controlled inducers of metabolic enzymes. Many substances induce hepatic enzymes (for example, phenytoin and some barbiturates), but they do not usually have therapeutic value owing to their other pharmacological side-effects. Particulate material, such as colloidal carbon, which can similarly induce metabolic enzymes following uptake into the liver, is retained in the body for long periods because it cannot be metabolized and thus is not used therapeutically.

Some PFCs can induce hepatic enzymes $^{6}$ without these side-effects. PFCs appear to have little or no pharmacological action and they are excreted (as vapour via the lungs) over a period of days following injection at a rate which depends primarily on the molecular mass of individual compounds ${ }^{2,3}$. The degree of enzyme induction by PFCs is related to their physico-chemical properties $^{7}$, and it should therefore be possible to use different PFC emulsions selectively to induce specific enzyme pathways. This could be of considerable therapeutic benefit, for example, in the treatment of paracetamol poisoning, where the production of toxic metabolites (produced via the mixed function oxidase system) could be eliminated if the glucuronidation pathway could be activated to a greater extent than the mixed function oxidase system.

Kenneth C. Lowe

Clive Washington*

Departments of Life Science

and *Pharmaceutical Sciences,

University of Nottingham,

University Park,

Nottingham NG7 2RD, UK

1. Otto, B. R., Venweij-van Vught, A. M. J. J. \& MacLaren D. M. Nature 358, 23-24 (1992).

2. Lowe, K. C. Vox Sang. 60, 129-140 (1991)

3. Lowe, K. C. Clin. Hemorheol. 12, 141-156 (1992)

4. Clark, L. C. \& Gollan, F. Science 152, 1755-1756 (1966).

5. Bentley, P. K. et al. J. Pharm. Pharmac. 41, 661-663 (1989).

6. Ravis, W. R., Hoke, J. F. \& Parsons, D. L. Drug Metab. Rev. 23, 375-411 (1991).

7. Lowe, K. C. \& Armstrong. F. H. Biomat. Art. Cells Immob. Biotech. (in the press).

\section{Decaying neutrino theory}

SIR - It is widely believed that Sciama's 1 decaying neutrino theory has been disproved by the failure of Davidsen $e t a l .^{2}$ to detect an unidentified emission line at $\sim 15 \mathrm{eV}$ from the dark matter in the cluster of galaxies A665. But recent X-ray data ${ }^{3}$ for A665 favour models in which the dark matter in the regions of the cluster observed by Davidsen et al. is mainly baryonic. So the decaying neutrino theory continues to be viable.

The X-ray data for A665 have been obtained by Hughes and Tanaka ${ }^{3}$ using

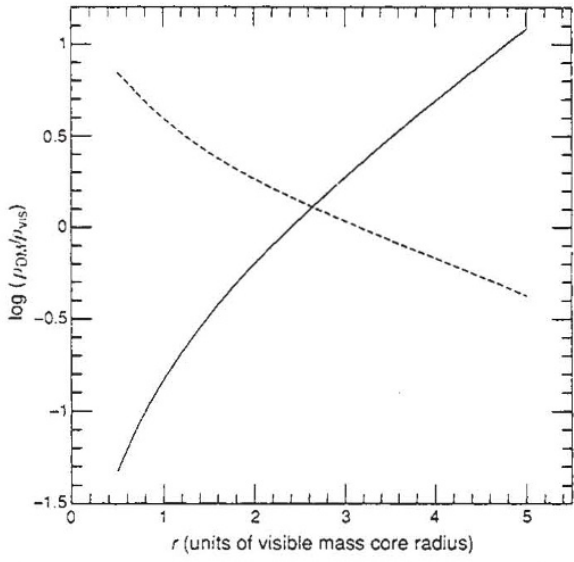

The local (diffuse dark)-to-visible density ratio as a function of a radius (in units of the scale-size, $\lambda$, of the visible mass distribution) between $1 / 2 \lambda$ and $5 \lambda$ for the spiral galaxy NGC 3198 (solid line; ref. 5) and for the cluster of galaxies A665 (dashed line; ref. 3 ). The scale-size $\lambda$ is equal to the disk exponential length for NGC 3198 and to 1.3 times the $\mathrm{X}$-ray core radius for $\mathrm{A} 665$.

the Ginga satellite. Combined X-ray and optical data have allowed them to construct a detailed self-consistent mass model of A665. This model includes: (1) a galaxy component of radial profile given by $\rho_{G} \sim\left(1+(r / 530 \mathrm{kpc})^{2}\right)^{-1}$; and (2) an X-ray emitting, hot gas component of radial profile $\rho_{X} \sim(1+(r / 380$ $\left.\mathrm{kpc})^{2}\right)^{-1}$. Their preferred resulting gravitational mass distribution, $\rho_{b} \sim(1+(r)$ $\left.298 \mathrm{kpc})^{2}\right)^{-1.36}$, implies the presence of a dark-matter component more concentrated than the two visible ones. From their mass model we compute a quantity $\gamma$, defined as the ratio between the mass in diffuse dark matter and the mass in gas and in galaxies (inclusive of their dark haloes). We then find that $\gamma$ goes from $\sim 11$ at $r=0.2 \mathrm{Mpc}$ to $\sim 2$ at $r=2$ Mpc. Thus in A665 the diffuse dark matter is more concentrated than the visible matter by an order of magnitude. More conclusively, the relative distribution of dark and luminous matter in clusters is opposite to what is found in galaxies ${ }^{4}$. In the well-studied exemplary case of the spiral galaxy NGC 3198, the dark-to-luminous mass ratio increases markedly going outwards in radius ${ }^{5}$. To highlight this dramatic difference, in the figure we plot the local dark-to-visible density ratio between one-half and five times the typical size of the visible matter distribution for A665 (dashed lines) and NGC 3198 (solid line), using the mass model solutions of refs 3 (already described) and 5, respectively. At the edge of the optical structure the dark-tovisible density ratio in galaxies is $\sim 10$ times bigger than in clusters, whereas at the centre it is $\sim 200$ times smaller than in clusters. This supports the view that the nature of dark matter is different in clusters and in galaxies.

A similar high concentration of dark matter at the centres of clusters has been derived for the Perseus ${ }^{6}$, Coma ${ }^{7}$ and A85 (ref. 8) and A2199 (ref. 8) clusters and from gravitationally lensed arcs in A370 (ref. 9) and other clusters. It has been argued ${ }^{6.7}$ that this concentration probably implies that the dark matter in these clusters is dissipative, and therefore baryonic.

We conclude that Davidsen et al.'s failure to detect an emission line from decaying neutrinos in the core of A665 cannot disprove in a straightforward way the decaying neutrino theory, in that the existence of substantial quantities of diffuse nonbaryonic dark matter in clusters has still to be confirmed.

\section{Dennis W. Sciama*}

\section{Paolo Salucci}

International School for Advanced Studies, and *International Centre

for Theoretical Physics,

Strada Costiera 11, 34014 Trieste, Italy

Massimo Persic

Osservatorio Astronomico di Trieste,

Via Tiepolo 11, 34131 Trieste, Italy

1. Sciama, D. W. Astrophys. J. 364, 549-554 (1990).

2. Davidsen. A. F. et al. Nature 351, 128-130 (1991)

3. Hughes, J. P. \& Tanaka, Y. Astrophys. J. 398, 62-68 (1992).

Persic, M. \& Salucci, P. Astrophys. J. 355, 44-51 (1990).

5. van Albada, T. S., Bahcall, J. N., Begeman, K. \& Sancisi, R. Astrophys. J. 295, 305-313 (1985)

6. Eyles, D. et al. Astrophys. J. 376, 23-32 (1991).

7. Briel, U. G.. Henry, J. P. \& Bohringer, H. Astr. Astrophys. 259, L31-L34 (1992).

8. Gerbal, D., Durret, F., Lima-Neto, G. \& Lachièze-Rey, M. Astr. Astrophys. 253, 77-88 (1992).

9. Bergmann, A. G., Petrosian, V. \& Lynds, R. Astrophys. J. 350, 23-35 (1990).

\section{Scientific Correspondence}

Scientific Correspondence is a relatively informal section of Nature in which matters of general scientific interest, not necessarily those arising from papers appearing in Nature, are published. Because there is space to print only a small proportion of the letters received, priority is usually given according to general interest and topicality, to contributions of fewer than 500 words, and to contributions using simple language. 